\title{
CRISPR/Cas9: at the cutting edge of hepatology
}

\author{
Francis P Pankowicz, ${ }^{1,2}$ Kelsey E Jarrett, ${ }^{3,4}$ William R Lagor, 1,3,4,5 \\ Karl-Dimiter Bissig ${ }^{1,2,5,6,7,8,9}$
}

Center for Cell and Gene Therapy, Center for Stem Cells and Regenerative Medicine, Baylor College of Medicine, Houston, Texas, USA

${ }^{2}$ Graduate Program Department of Molecular \& Cellular Biology, Baylor College of Medicine, Houston, Texas, USA

Department of Molecular Physiology and Biophysics, Baylor College of Medicine, Houston, Texas, USA ${ }^{4}$ Integrative Molecular and Biomedical Sciences Graduate Program, Baylor College of Medicine, Houston, Texas, USA ${ }^{5}$ Texas Medical Center Digestive Diseases Center, Baylor College of Medicine, Houston, Texas, USA

${ }^{6}$ Graduate Program in Translational Biology and Molecular Medicine, Baylor College of Medicine, Houston, Texas, USA

${ }^{7}$ Department of Molecular and Cellular Biology, Baylor College of Medicine, Houston, Texas, USA

${ }^{8}$ Program in Developmental Biology, Baylor College of Medicine, Houston, Texas, USA ${ }^{9}$ Dan L. Duncan Cancer Center, Baylor College of Medicine, Houston, Texas, USA

Correspondence to Dr Karl-Dimiter Bissig, Department of Molecular and Cellular Biology, Baylor College of Medicine, 1, Baylor Plaza, Houston, TX 77030-3411, USA; bissig@bcm.edu

Received 5 April 2017 Accepted 7 April 2017 Published Online First 5 May 2017

CrossMark

To cite: Pankowicz FP, Jarrett KE, Lagor WR, et al. Gut 2017:66:1329-1340.

\begin{abstract}
Clustered Regularly Interspaced Short Palindromic Repeats (CRISPR)/Cas9 genome engineering has revolutionised biomedical science and we are standing on the cusp of medical transformation. The therapeutic potential of this technology is tremendous, however, its translation to the clinic will be challenging. In this article, we review recent progress using this genome editing technology and explore its potential uses in studying and treating diseases of the liver. We discuss the development of new research tools and animal models as well as potential clinical applications, strategies and challenges.
\end{abstract}

\section{INTRODUCTION}

Clinical application of gene therapy-the transfer of a wild-type allele to the human patient to replace a mutated gene ${ }^{1}$ - has proven very challenging for several reasons, including inefficient delivery into the target cell, the need for sustained expression of the transgene, and deleterious patient immune response. The first gene therapy trial ${ }^{2}$ sought to correct adenosine deaminase deficiency by transferring a copy of the wild-type gene into haematopoietic stem cells ex vivo and re-infusing the cells into the patient. This trial demonstrated the feasibility of gene therapy, which prompted a flurry of trials in the biomedical community. A few years later, a fatal systemic inflammatory response occurred with a liver-directed adenoviral vector ${ }^{3}$ and it became clear that retroviral integration could result in unexpected neoplasias. ${ }^{4}$ While many of these initial setbacks have been overcome by improvements in vector design and cell-based therapy procedures, there is still considerable room for improvement. Recent advances in genome editing are driving a fundamental paradigm shift from overexpression of defective gene products to precisely modifying a patient's own DNA. The notion of treating disease by removing or repairing harmful mutations is a tantalising one, and may be a solution to the many disorders not amenable to pharmacological treatment.

Genome editing has been attempted for some time, but the complexity of zinc finger nucleases, combined with the secrecy of proprietary technology, delayed further development. Later, Transcription Activator-Like Effector Nuclease technology became available, and genome editing started to gain momentum. Both technologies had one major drawback: the nucleases used to cut DNA were inefficient. This changed with the development of Clustered Regularly Interspaced Short Palindromic Repeats (CRISPR)/Cas9 genome editing: this technology is more efficient than previous generations of designer nucleases, and it has the added benefit of being simple to use, from design to execution. Many physicians and scientists are now searching for the best clinical applications for this promising technology.

The liver has several advantages over other organs for somatic genome editing for both hepatic disorders and for systemic metabolic conditions triggered by a mutated or dysregulated gene expressed in the liver. First, the liver is an immuneprivileged organ and favours immune tolerance over induction of immunogenicity. ${ }^{5}$ Second, many gene therapy vectors, including nanoparticles, have a natural tropism towards the liver, which should help to reduce the risk of a severe immune response (see below). Third, the 'exit strategy' in the liver is more favourable than in other organ systems such as the brain or heart, so if CRISPR/ Cas9-mediated genome editing leads to deleterious complications such as neoplastic growth, the problematic area could be more readily resected. Such an outcome is of course not desirable, but must be carefully weighed against the potential benefits to patients when introducing CRISPR/Cas9 into the clinic.

Here, we will discuss how CRISPR/Cas9 is used in research as well as its potential clinical applications. We will explain the benefits of this technology as well as discuss the major hurdles involved in translating it to the clinic.

\section{CRISPR/Cas9 genome editing}

The CRISPR/Cas9 genome editing system is derived from a naturally occurring antiviral immune system found in many species of bacteria. The first discovery came in 1987, when Ishino et $a l^{6}$ noticed a cluster of repeat sequences, interrupted by variable spacer sequences, later referred to as CRISPR. ${ }^{7}$ However, it was not until 2005 that these spacer sequences were recognised as foreign in origin ${ }^{8-10}$ and postulated to play a role in host adaptive immunity. ${ }^{8}$ This defence mechanism relies on a family of CRISPR-associated (cas) genes. ${ }^{711} 12$ The Cas9 gene encodes an RNA-guided nuclease that normally protects the host from phage infection through sequence-specific destruction of foreign DNA. ${ }^{13}{ }^{14}$ Years of work by several groups finally culminated in the identification of all key components of a recombinant CRISPR/Cas9 system (box 1) and the demonstration of its functional capability in mammalian cells. ${ }^{15-18}$

The mechanism of CRISPR/Cas9 activity consists of a few essential components (figure 1A): a processed spacer/repeat sequence that is transcribed to generate the 'crRNA', ${ }^{19}$ which binds to a separately transcribed and partially complementary 'tracrRNA'. ${ }^{14}$ Together, these RNAs are able to guide the Cas 9 protein to a target site, defined by 


\section{Box 1 Glossary}

Cas9: CRISPR-associated protein 9, an endonuclease from bacteria that forms a ribonucleoprotein with the sgRNA, which can be directed to cause a double-strand break at most variable $\sim 20$ base pair (bp) DNA sequences via sgRNA target sequence. sgRNA: single guide RNA. An artificial chimaera of crRNA and tracrRNA, the two bacterial RNA components that direct Cas9 to DNA sequences for cleavage. The first $\sim 20$ bp of sgRNA (or crRNA) are variable and complementary to the target site. PAM: protospacer adjacent motif. The sequence required immediately downstream of the target sequence. The PAM varies depending on the bacterial origin of the Cas9 protein. DSB: double-strand break. CRISPR/Cas9 introduces a blunt DSB in the target DNA three bps upstream of the PAM.

NHEJ: non-homologous end joining. A method of DSB repair that does not use a template strand, and which can result in the introduction of insertions or deletions of variable length at the cut site.

$H D R$ : homology-directed repair. A repair mechanism using a DNA template to repair double-stranded DNA breaks via homologous recombination.

CRE-loxP technology: a method of inducing site-specific recombination between two sites (LoxP sites), which is catalysed by the Cre recombinase protein. This phage recombination system is the 'gold standard' of creating conditional knockout alleles in mouse transgenesis.

Zygote: a fertilised egg, which is still at the single-cell stage. The genome of the zygote is amenable to editing by injected CRISPR/Cas9 molecules. Modifications in zygotes are then carried on in all subsequent cell divisions during embryonic development.

complementarity to the spacer sequence, in combination with a species-specific protospacer adjacent motif (PAM), which must be present immediately following the target site for DNA cleavage to occur. ${ }^{20} 21$ This mechanism was further simplified in 2012, when Jinek et $a l^{22}$ showed that a chimaeric crRNA: tracrRNA transcript, termed 'single guide RNA' (sgRNA), is equally capable of directing Cas9 to its target sequence to cut DNA. The Cas9/sgRNA complex surveys the genome for complementary sites based on Watson-Crick base pairing between the guide sequence and DNA. On successful recognition of the target, Cas9 undergoes a conformational change that engages its two nuclease domains. ${ }^{23-25}$ The nuclease domains cleave both strands of the target DNA at approximately -3 nucleotides before the PAM, generating a double-strand break (DSB). ${ }^{26}$ Single amino acid mutations in either nuclease domain of Cas9 result in a 'nickase', ${ }^{27}$ which produces single-stranded DNA nicks rather than DSBs. Likewise, mutations of both nuclease domains result in a version of Cas9 that can survey and bind to DNA, but that is incapable of cutting. This catalytically inactive or 'dead' Cas9 (dCas9) can be modified with fusion proteins to activate or repress transcription ${ }^{28-31}$ or change the epigenetic status of the local chromatin. ${ }^{32-35}$ However, it is the ability of Cas9 to efficiently and easily generate DSBs in DNA that has opened the door for a multitude of basic research and therapeutic applications.

The CRISPR/Cas9 system is the most efficient and flexible genome editing system to date, requiring only customisation of the sgRNA to generate DSB at a user-designated site. The PAM for the most commonly used Streptococcus pyogenes Cas9 (SpCas9) is ' $\mathrm{NGG},{ }^{20}$ which occurs frequently in the genome and generally imposes only minimal constraints on target site selection and sgRNA design. The second most commonly used Cas9 ortholog from Staphylococcus aureus has a more restrictive 'NNGRRT' PAM, ${ }^{36}$ although recent efforts have been successful in partially re-engineering Cas9 PAM specificities through rational design. ${ }^{37} 38$

Once the Cas9 has bound and cleaved the DNA, the host's DNA repair machinery repairs the DSB by either homologous recombination (homology-directed repair (HDR)) or nonhomologous end joining (NHEJ) (figure 1B). HDR uses a template strand to precisely repair a DSB, such that no mutations are introduced at the cleavage site. While this occurs naturally during the cell cycle using the sister chromatid as repair template, ${ }^{39-41}$ exogenous template DNA can also be introduced for integration based on homology to the target site. This process is dramatically more efficient for insertion of foreign genetic material at sites when Cas9 is used to introduce targeted DSBs. While HDR has been achieved for years with conventional gene targeting in mouse embryonic stem cells, CRISPR/Cas9 makes this feasible in most dividing cells.

NHEJ is the dominant DSB repair pathway in most mammalian cells and organisms, and is active in all phases of the cell cycle. As the name suggests, this pathway does not require a homologous template, and can repair irregular breaks that have overhanging strands such as those that often result from exogenous DNA-damaging agents. In contrast to HDR, NHEJ can result in genomic insertions and/or deletions (referred to as 'indels') during processing of the ends of the $\mathrm{DSB}^{42}$ (figure $2 \mathrm{~A}$ ). These indels are of varying size and, when targeted within protein coding sequences, can create inactivating missense or nonsense mutations. Most indels introduced by Cas9 cleavage are small frameshift mutations (usually \pm 1 or 2 bases). Frameshift mutations that occur before the penultimate exon in a protein-coding gene usually result in nonsense-mediated decay (NMD) of the mutant transcript, effectively 'knocking out' the gene. When multiple sgRNA are used in combination, the NHEJ pathway can also be used to delete intervening DNA sequences.

Both DSB repair pathways are used in parallel by a single cell and have their own potential utilities and pitfalls. HDR is primarily active during the $S$ and G2/M phases, while cells in G0/ G1 almost exclusively repair DSB by NHEJ. ${ }^{39-41}$ In addition, there is evidence that even during $S$ phase, when HDR is most likely to occur, NHEJ is still the favoured repair mechanism in human cells. ${ }^{39}$ The mechanism of DSB repair is important to consider when designing CRISPR/Cas9 strategies in quiescent cells such as hepatocytes.

In the following sections, we will discuss how CRISPR/Cas9 has facilitated research in the field of hepatology, primarily as it pertains to human liver disease. We will also discuss potential clinical applications of CRISPR/Cas9 for liver disease and disorders, which might benefit from hepatic genome editing strategies.

\section{CRISPR/Cas9 preclinical and research applications} In vitro applications

A major milestone in the development of CRISPR/Cas9 was its adaptation for use in mammalian cells, ${ }^{17}{ }^{18}$ which provided researchers with a powerful tool to study genetic perturbations in tissue homeostasis and disease. Since then, a significant body of work has used CRISPR/Cas9 in the tissue culture setting to explore various aspects of liver biology. While these studies are 
Figure 1 CRISPR/Cas9 structure and simplified repair mechanism. (A) The molecular elements of CRISPR/Cas9. The bacterial Cas9 protein that binds to two different single-stranded RNAs (crRNA and tracrRNA), which are partially complementary in bacteria but have been combined as sgRNA for research purposes. The species-dependent protospacer adjacent motif (PAM) must be present immediately following the guide sequence for DNA cleavage to occur. (B) Repair mechanism in mammalian cells induced by CRISPR/Cas9-mediated DNA cleavage (simplified).

It is important to note that perfectly repaired DNA will be repeatedly cut until the targeting sequence is modified. See text for more details on non-homologous end joining (NHEJ) and homology-directed repair (HDR). Red arrow is the location within the target sequence where the double-strand break takes place.

A

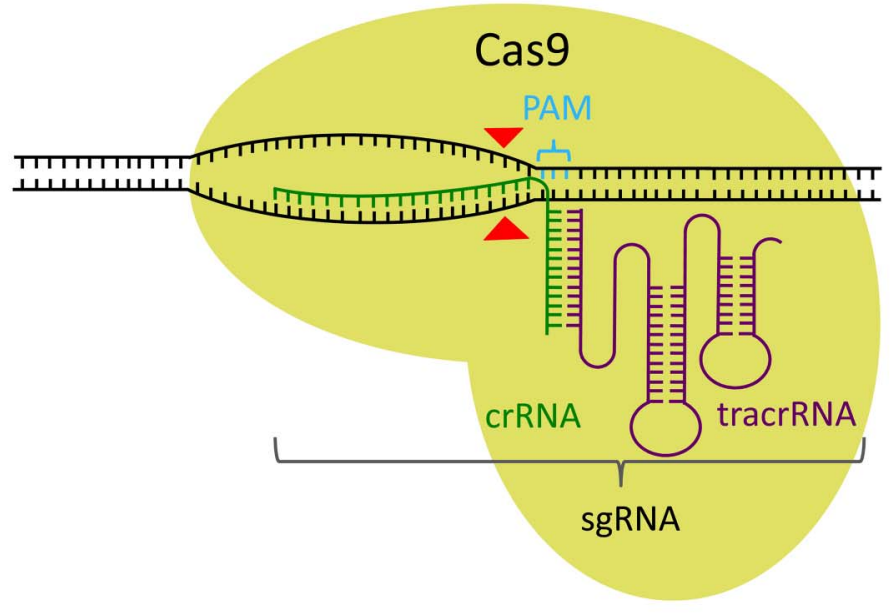

B

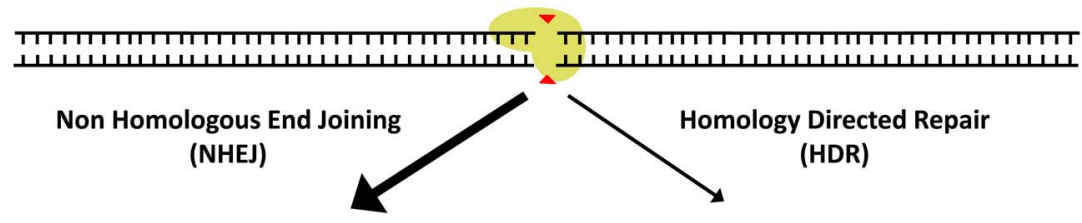

Perfect repair (religation)

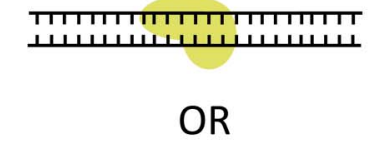

Variable length insertions

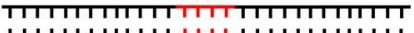

\section{OR}

Variable length deletions

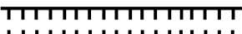

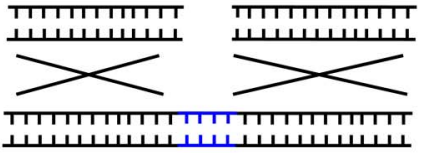

Exogenous donor template-dsDNA

OR

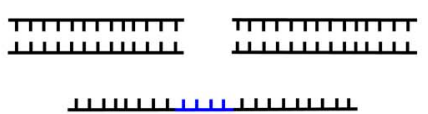

Exogenous donor template - ssDNA diverse and involve many genetic targets, there are common underlying themes and strategies.

So far, most efforts have been focused on either deleting a gene by NHEJ or introducing a transgene by homologous recombination. When knocking out a gene, there are several potential strategies. The most common method is to use a single sgRNA targeted to a critical exon in order to introduce indels, likely causing a frameshift mutation and/or NMD (figure 2A). This approach has been used to validate, in vitro, potential oncogenes discovered in human liver cancers, such as acid sensing ion channel $1 \mathrm{a}^{43}$ and eukaryotic elongation factor 2 kinase. $^{44}$ Similarly, isocitrate dehydrogenase 1 , commonly mutated in intrahepatic cholangiocarcinoma, ${ }^{45}$ has been deleted in the HepG2 cell lines to dissect its biological and metabolic functions. ${ }^{46}$

To increase the chances of gene deletion, several groups have used multiple sgRNAs targeting different regions of the gene of interest simultaneously. For instance, the aspartate $\beta$-hydroxylase gene has been targeted by three separate sgRNAs to generate a HepG2 knockout line. ${ }^{47}$ CRISPR/Cas9-induced NHEJ can also be used for the deletion of entire genomic regions; when two DSBs are induced at the same time in close proximity to each other, it is likely that the intervening region will be lost during
NHEJ repair ${ }^{27} 4849$ (figure 2C). There are several advantages to this research design. For instance, Pankowicz et al ${ }^{50}$ has shown that whole exons can be removed without the risk of unpredictable indels in the reading frame of the hydroxyphenylpyruvate dehydrogenase (HPD) gene. This deletion strategy can also be used to delete a specific enhancer region, as has been done in the cytochrome P450 (CYP) 2D6 (CYP2D6) promoter of HepG2 cells ${ }^{51}$ (figure 2D). Similarly, Hep3B cells have been modified using CRISPR/ Cas9 to delete the zic family member 2 binding region in the octamer binding protein 4 promoter. $^{52}$

CRISPR/Cas9-induced DSB can also be used to increase the rate of HDR, which is particularly useful for introducing transgenes at virtually any desired locus (figure $2 \mathrm{~B}$ ). This approach is being broadly used in biomedical science, particularly to generate reporter cell lines for screens. ${ }^{17} 5354$ The strategy has also been used for in vitro lineage tracing during bile duct development. In this case, the fluorescent reporter mCherry, and the selection marker puromycin $\mathrm{N}$-acetyl-transferase, were knocked into the cytokeratin 7 locus in human induced pluripotent stem cells. ${ }^{55}$ CRISPR/Cas9-assisted HDR has also been used in a potential therapeutic setting for the urea cycle disorder arginase 1 deficiency. ${ }^{56}$ 
Figure 2 Genome editing approaches for biomedical applications. Examples of genetic modifications are given. For details, see 'CRISPR/Cas9 preclinical and research applications' or 'Medical applications for CRISPR/Cas9 in the liver' section. HDR, homology-directed repair; NHEJ, non-homologous end joining.

\section{Exon mutation (NHEJ)}

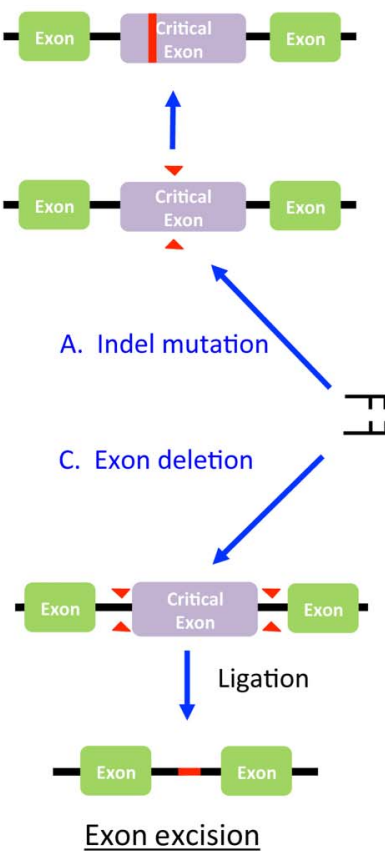

Targeted gene addition (HDR) Correction (HDR)
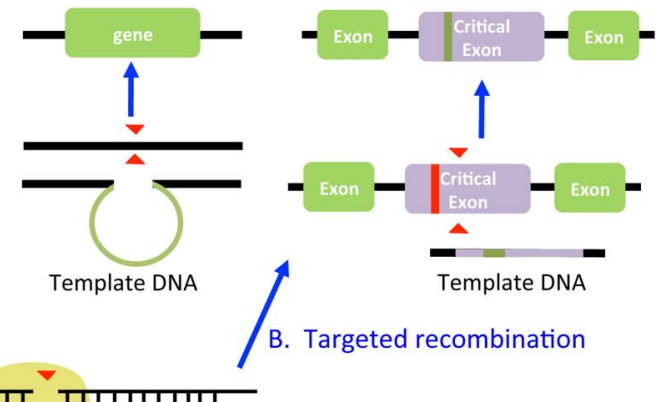

Targeted recombination
Finally, the nuclease domain of Cas9 can be inactivated (dCas9), and other functional domains can be attached. ${ }^{28-35}$ This technology has been used to characterise the metformin response in the liver. In one study, metformin responsive enhancers were identified, and targeted with a dCas9-VP64 fusion protein to measure the transcriptional response in the Huh7 cell line. ${ }^{57}$ Activation of genes in this region via the CRISPR-guided VP64 transactivator showed significantly increased levels of ataxia telangiectasia mutated (ATM), exophilin 5, DEAD-Box Helicase 10 mRNA, suggesting a possible role for these proteins in the mechanism of action of metformin.

\section{In vivo applications}

Somatic genome editing using CRISPR/Cas9

As scientists move from cell culture to in vivo systems, delivery of macromolecules, such as the CRISPR/Cas9 machinery, becomes a limiting factor. However, the field of hepatology has greatly benefited from the development of an in vivo transfection method called hydrodynamic tail vein injection (HTVI), which is a simple and efficient way to deliver DNA into murine hepatocytes. ${ }^{58} 59$ The exact nature of the uptake is not known, but up to $40 \%$ of hepatocytes can be transfected with this method. Hydrodynamic tail vein injection has already proven very useful for a variety of CRISPR/Cas9 applications.

Several groups have used CRISPR/Cas9 editing to promote tumour growth or study tumour suppressor candidate genes in the liver. ${ }^{60-62}$ For instance, Xue et a $l^{60}$ targeted the tumour suppressors Pten and p53 alone and in combination using HTVI. Each sgRNA was able to create indels and disrupt its respective gene, although with relatively low frequencies (2\%-7\%). While the individual gene mutations did not generate tumours within the study's 3-month timeframe, combining Pten and p53 sgRNAs was capable of generating liver tumours similar to those of transgenic animals with CRE-loxP-deleted Pten and p53. In addition, they generated a gain-of-function mutation in $\beta$-catenin by targeting the Ctnnb1 gene with sgRNAs and providing a single-stranded DNA template for HDR. This template was successfully integrated in only $\sim 0.5 \%$ of hepatocytes, as measured by nuclear localisation of $\beta$-catenin. This study highlights several points to consider when using CRISPR/Cas9 for somatic genome engineering in the liver. First, it demonstrates the ease of bypassing the lengthy process of embryonic stem cell targeting with CRE-loxP technology. This is tempered by the low rate of indel formation, but this is not limiting in a positive selection setting such as expansion of liver cancer. Furthermore, it demonstrates the considerably lower rates of HDR compared with NHEJ in the liver. Another study using multiple sgRNA in a sleeping beauty transposon cassette demonstrated development of hepatocellular carcinoma (HCC) and intrahepatic cholangiocarcinoma by deleting several tumour suppressor genes in parallel. ${ }^{61}$ This study revealed a high $(79 \%)$ incidence of biallelic mutations within the multiple sgRNA target sites, confirming the high efficiency of NHEJ in hepatocytes. Another recent study used a genome-wide sgRNA library to identify new liver tumour suppressors in transgenic $p 53^{-1-}$ and myc overexpressing embryonic liver progenitors injected subcutaneously. ${ }^{62}$ In a second step, they validated the tumour suppressor neurofibromatosis type 1 using somatic genome editing in the murine liver.

Somatic editing with CRISPR/Cas9 has also been successfully used to explore experimental therapies for liver disease. ${ }^{50}$ 63-65 Hereditary tyrosinemia type I (HT-I) is caused by a defect in the fumarylacetoacetate hydrolase $(f a b)$ gene, which leads to a toxic accumulation of tyrosine catabolites in the liver. Yin et al used HTVI to transfect livers of $f a b^{-1-}$ mice with sgRNA, Cas9 and single-stranded DNA templates. ${ }^{65}$ They observed an initial HDR editing efficiency of $0.4 \%$ of hepatocytes. However, these edited hepatocytes displayed a growth advantage over non-edited hepatocytes and eventually expanded up to $33 \%$ of the liver, improving the weight loss phenotype over the 30-day observation period. In a follow-up study using nanoparticles to deliver Cas9 and adeno-associated virus (AAV) for the sgRNA and template DNA, they found a higher initial editing rate of up to $6 \% .^{64}$ Yang et al explored a dual AAV CRISPR/Cas9 approach in the $s p f^{a s h}$ mice, a murine model for ornithine transcarbamylase deficiency. Mice injected as neonates showed correction in $10 \%$ of hepatocytes, which improved survival when challenged with a 
high-protein diet, but the same therapeutic approach in adult mice was much less successful $(<1.7 \%$ correction). Furthermore, deep sequencing revealed very high frequencies (up to $50 \%$ adult, $35 \%$ neonate) of indels in the sgRNA target site, consistently exceeding the correction rate in all mice analysed. These studies indicate that HDR can be achieved in the settings of growth during early development, as well as in metabolic disease models with regenerating hepatocytes. However, it should be noted that alleles that do not undergo HDR are very likely to get NHEJ-induced mutations. This phenomenon is especially important to consider for compound heterozygotes, where unintended NHEJ editing of the other allele could generate more deleterious mutations.

Some disorders will be amenable to an approach called 'metabolic pathway reprogramming', which avoids generating potentially dangerous mutations in the diseased gene (figure 3). This approach deletes a disease pathway-associated gene in order to reroute metabolic pathways and improve a metabolic condition. As a proof-of-principle, we applied this strategy to $\mathrm{fab}^{-/-}$mice and were able to rescue the lethal phenotype with a one-shot therapy targeting the hydroxyphenylpyruvate dioxygenase $(h p d)$ gene, which acts upstream of fah. By deleting critical $h p d$ exons with two separate sgRNAs, we safely inactivated $h p d$ in $\sim 8 \%$ of hepatocytes following hydrodynamic delivery. Double mutated hepatocytes $\left(\mathrm{fah}^{-/-} / \mathrm{hpd} \mathrm{d}^{-/-}\right)$had a growth advantage and almost completely repopulated the liver over the course of 2 months. In a similar approach, Liang $e t \mathrm{al}^{66}$ deleted the Fas receptor and were able to demonstrate resistance to concanavalin A-induced fulminant hepatic failure.

Finally, Jarrett et $a l^{67}$ recently demonstrated that somatic genome editing with CRISPR/Cas9 can be used for disease modelling. Using AAV to deliver sgRNA targeting the low-density lipoprotein receptor $(l d l r)$ to Cas9 transgenic animals, a novel model for familial hypercholesterolaemia was established. The nearly complete removal of hepatic Ldlr protein produced a severe hypercholesterolaemia and atherosclerotic plaque. They further showed that dual AAV vectors could be used for concomitant disruption of apolipoprotein B $(a p o B)$, which dramatically reduced plasma cholesterol levels and offered complete

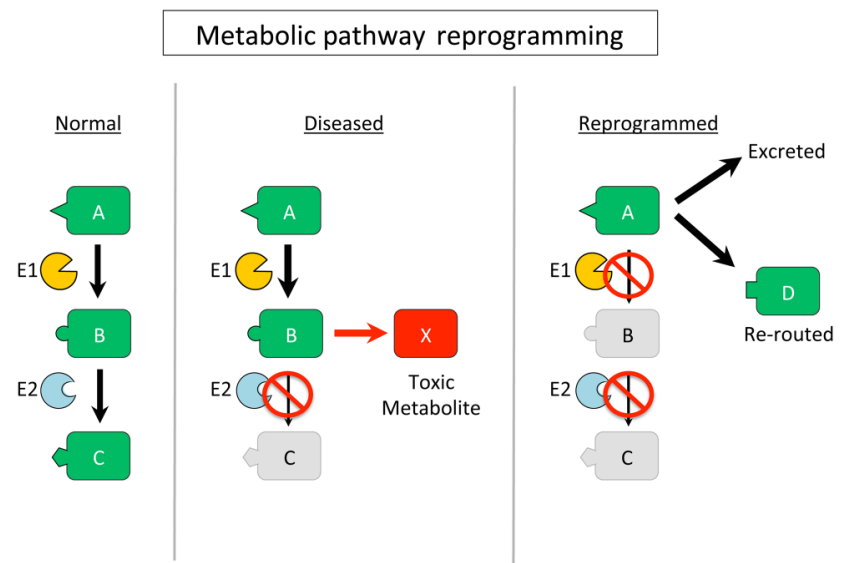

Figure 3 Schematic representation of metabolic pathway reprogramming within a single pathway. In a healthy individual, the metabolic pathway proceeds as normal, with functional clearance of intermediate metabolites. Toxic accumulations in a diseased state occurs due to a non-functional or hypomorphic downstream enzyme (E2), which can be alleviated by using CRISPR/Cas9 to delete a wild-type enzyme (E1) in a disease-associated pathway leading to a more benign phenotype. protection from atherosclerosis. Besides gaining new insight into familial hypercholesterolaemia and apoB as a therapeutic target, this model establishes a new method to efficiently knockout genes in the liver without the need for laborious germline targeting.

\section{Zygote injection of CRISPR/Cas9}

CRISPR/Cas9 has revolutionised the generation of genetically modified organisms. Previously, gene targeting was accomplished by first modifying embryonic stem cells, followed by blastocyst injection and screening for germline transmission in offspring. ${ }^{68}$ This process was inefficient, time-consuming and expensive, limiting the rate at which animal research could be performed. In 2013, several groups reported that zygote injection with CRISPR/Cas9 could lead to efficient gene knockout at several loci in zebrafish, ${ }^{69-71}$ mice, ${ }^{72}{ }^{73}$ rats $^{74}{ }^{75}$ and rabbits, ${ }^{76}$ completely bypassing the need for targeting in embryonic stem cells. Since then, several groups have used CRISPR/Cas9 to generate knockout models of animals in order to study hepatic gene function. In zebrafish, this method has been used to study hepatic glucose metabolism on deletion of both the leptin ${ }^{77}$ and insulin receptor. ${ }^{78}$

In mice, zygote injection of CRISPR/Cas9 has been used to generate models for the study of lipid disorders. For example, the E167K mutation of the transmembrane 6 superfamily member 2 gene (TM6SF2) had been previously correlated with lower total plasma cholesterol and low-density lipoprotein (LDL) cholesterol, but increased susceptibility to non-alcoholic fatty liver disease. ${ }^{79}{ }^{80} \mathrm{Fan}$ et $\mathrm{al}^{81}$ investigated the long-term consequences of TM6SF2 deletion in mice, using a CRISPR/ Cas9-generated knockout model. In a separate study, Nakagawa et $a l^{82}$ used CRISPR/Cas9-injected zygotes to generate a conditional knockout (CRE-loxP technology) model for cAMP responsive element binding protein 3-like 3 (creb3l3). Generation of these mice was an impressive use of HDR in zygotes through simultaneous insertion of loxP sites with donor oligos, however, with a very low efficiency (1 mouse of 277 injected zygotes). In order to elucidate the role of the protein in the liver and intestine, mice were crossed with either albumin-Cre (liver) or villin-Cre (intestine) transgenic mice. Only hepatic deletion of the CREB3L3 resulted in increased plasma lipid and cholesterol content. Interestingly, this is a deviation from the global CREB3L3 knockout, which results in hypocholesterolaemia.

Rats have also been modified using CRISPR/Cas9 and have notable differences from their mouse counterparts. For example, a rat model of HT-I generated by zygote injection of CRISPR/Cas9 displayed all of the hallmarks of the disease, including hypertyrosinemia, liver failure and renal tubular damage. Importantly, these rats also developed cirrhosis and fibrosis, which occur in affected humans but no other animal models of the disease. ${ }^{83}$ Recently, two other rat models for cytochrome metabolism have been generated using a CRISPR/Cas9 zygote injection strategy. Both CYP2E $1^{84}$ and CYP3A1/2 $2^{85}$ knockout rats have been successfully generated by targeting critical exons of the according genes. In both cases, rats were demonstrated to be viable and fertile, with drug metabolism profiles consistent with successful gene knockout.

To study human drug metabolism in mice, Barzi et al recently generated a novel mouse model that used zygote injections to simultaneously knockout three genes: fah, common gamma chain of IL-2 receptor and the recombination activating 2 gene. The injected zygotes were homozygous for a conditional knockout (CRE-loxP) allele of the P450 oxidoreductase $(p o r) .{ }^{86}$ Por is 
the only electron donor for all P450 cytochromes and a deletion results in a functional inactivation of the whole cytochrome superfamily. The resulting mice can be repopulated with human hepatocytes, and on deletion of the murine por, were found to demonstrate human-like drug metabolism. This next generation humanised mouse shows the power of CRISPR/Cas9 for simultaneously targeting multiple genes on existing complex genetic backgrounds.

In addition, CRISPR/Cas9-generated knockout models generally avoid the need for selection cassettes, which have been shown to interfere with cellular gene regulation and local gene expression. ${ }^{87-89}$ Although murine zygote injections are believed to have a lower CRISPR/Cas9-induced off-target mutation rates than human cell lines, ${ }^{48} 9091$ it is advisable to backcross offspring with wild-type mice to eliminate potential confounding off-target mutations. For generation of knockout models, CRISPR/Cas9 zygote injection has essentially replaced classical gene targeting in mouse embryonic stem cells, and might ultimately supplant targeting in embryonic stem cells for conditional alleles and targeted knockins. Due to the rapid success of this technology, we are likely to see many more applications for hepatic research soon.

\section{Medical applications for CRISPR/Cas9 in the liver}

CRISPR/Cas9 technology has revolutionised science and has the potential to transform medicine too. This has also been recognised outside of academia by commercial entities developing CRISPR/Cas9 products. However, as far as we know, there are currently no clinical trials ongoing for liver disease using CRISPR/Cas9 technology. The following section outlines some strategies for medical applications in the field of hepatology, while the major translational challenges are addressed in a separate section. We will only describe somatic editing approaches in the liver, since germline manipulations have important ethical, regulatory and societal implications that require further discussion.

\section{Deletion of dominant disease-causing alleles}

Current CRISPR/Cas9 technology is more effective at disrupting or removing target genes than correcting mutations. Therefore, mutated genes of the liver that have a detrimental effect in the liver or other organs are likely the lowest hanging fruit for therapeutic application of CRISPR/Cas9. For instance, familial transthyretin (TTR) amyloidosis is an autosomal dominant disorder presenting with varying degrees of neuropathy, cardiopathy, GI impairment and ocular depositions. The extracellular deposition of TTR-derived fibres in all affected organ systems is responsible for this heterogeneous disorder. In theory, this condition could be treated by the removal of the mutated allele. The allele could be deleted by several CRISPR-mediated strategies, each of which has unique advantages and caveats (figure 2).

The successful clinical application of a somatic genome editing approach using CRISPR/Cas9 technology will depend on several general and disease-specific factors. For instance, it is essential to know where the mutated protein is expressed and to what extent hepatic production contributes to disease pathology. It is also important to know what percentage of edited alleles will alleviate symptoms (ie, the threshold of correction). Clinical studies or case reports from orthotopic liver transplantations (OLT) can help to guide the selection of targets for diseases where patients are most likely to benefit.
Patients with TTR amyloidosis and severe polyneuropathy undergo therapeutic OLT ${ }^{92}$; however, patients with cardiomyopathy may experience accelerated disease progression after OLT due to deposition of wild-type TTR on a template of amyloid derived from the mutated TTR. ${ }^{93} 94$ Thus, in theory more patients could benefit from somatic genome editing in the liver than OLT. A gene editing approach might also be applied to fusion proteins resulting from translocations, such as the DNAJB1-PRKACA chimaeric transcript in fibrolamellar HCC. ${ }^{95}$ In this case, careful design of the CRISPR/Cas9 vectors would be needed to target only the precise breakpoint, while avoiding other alleles of the genes involved in the translocation.

\section{Deletion of foreign DNA and/or associated host factors}

CRISPR/Cas9 has recently been explored for its therapeutic potential in targeting human pathogens, particularly viral infections with double-stranded DNA intermediates such as papillomavirus $^{9697}$ and Epstein-Barr virus. ${ }^{98} 99$

Notably, HBV has emerged as an attractive target for CRISPR/Cas9 in the laboratory and, potentially, the clinic. HBV is a partially double-stranded DNA virus that infects primate and human hepatocytes at least partially by using the sodiumtaurocholate cotransporting polypeptide (NTCP) as a receptor. ${ }^{100}$ HBV establishes covalently closed circular DNA (cccDNA) within hepatocytes, which is maintained episomally in the nucleus and mediates chronic infection. This DNA replicative intermediate is an attractive target for the clinical use of CRISPR/Cas9.

Several groups have demonstrated targeted mutation of HBV in both the cell culture and animal model settings. Lin et al ${ }^{101}$ were the first group to use CRISPR/Cas9 to target HBV. Using cotransfection of Huh7 cells with sgRNA pairs and an HBV expressing vector, they were able to achieve up to $96 \%$ suppression of intracellular hepatitis B surface and core antigens. Using a HTVI model of HBV replication in mice, they were also able to show in vivo editing of $\mathrm{HBV}$ in about $5 \%$ of the DNA copies. A variety of groups have since reported similar findings, targeting various regions of the HBV genome. ${ }^{102-109}$ In addition, Kennedy et $a l^{102}$ also showed a synergistic effect between targeting HBV with CRISPR/Cas9 and using traditional antiviral therapies. It is important to note that HBV infects only humans and chimpanzees. Mouse models for $\mathrm{HBV}$ infection do not recapitulate the formation of cccDNA in hepatocytes, which is a target for therapy in humans. Dong et al ${ }^{103}$ used CRISPR/Cas9 targeting in an in vivo model for cccDNA expression ${ }^{110}$ to show a modest decrease in cccDNA concentration within the liver and a corresponding decrease in serum levels of the viral hepatitis $\mathrm{B}$ surface antigen and hepatitis B e antigen. Human liver chimaeric mice replicate $\mathrm{HBV}^{111} 112$ and may be the best model to demonstrate the effectiveness of a CRISPR/Cas9 antiviral approach.

In addition to DNA viruses, it has recently been proposed that RNA viruses might be targeted with CRISPR/Cas9 as well. The Cas9 variant from Francisella novicida (FnCas9) is reported to be capable of targeting mRNA from $\mathrm{HCV}^{113}$ Price et al developed FnCas9 to target both the positive and negative strands of HCV, and were able to inhibit HCV protein expression in a cell culture model of HCV infection. However, the same effect was observed when the nuclease domain was inactivated, suggesting that simply binding to the RNA was sufficient to interfere with translation. Interestingly, FnCas9 is also able to target DNA for double-strand cleavage similar to SpCas9, indicating the possibility for a dual targeting strategy in the future.

Aside from targeting hepatotropic viruses, CRISPR/Cas9 could be used to target host factors that are essential for viral 
replication but do not result in a severe hepatic phenotype. For instance, targeting the NTCP receptor to inhibit HBV entry would be a possibility, especially considering that the liver specific-knockout model has a surprisingly moderate phenotype in mice. ${ }^{114}$

\section{Substrate reduction approach}

A CRISPR-mediated approach to substrate reduction may be therapeutic when essential liver-produced proteins and metabolites lead to harmful effects even in the absence of a specific monogenic disorder, for instance, in hyperglycaemia and hyperlipidaemia. The liver has not been fully exploited in regard to antidiabetic therapies ${ }^{115}$ and somatic genome engineering opens many new therapeutic approaches towards lowering blood glucose levels.

The liver is also a key organ in lipid and lipoprotein metabolism. ${ }^{116}$ It is the site of synthesis and secretion of very lowdensity lipoprotein (VLDL) particles that carry triglyceride, cholesterol and fat-soluble vitamins to peripheral tissues. VLDL contain the apoB-100 (APOB) protein as their primary structural component, and are catabolised in the circulation to produce LDL. VLDL and LDL particles are both cleared primarily by the liver through the action of the LDLR. ${ }^{117} \mathrm{~A}$ substrate reduction approach using CRISPR/Cas9 could be pursued for $A P O B$, which is currently targeted by antisense oligonucleotides in severe cases of homozygous familial hypercholesterolaemia. ${ }^{118}$ However, careful design would be needed to avoid mechanism-related hepatic fat accumulation with $A P O B$ disruption.

Another attractive target for somatic editing in the liver is proprotein convertase subtilisin/kexin type 9 (PCSK9), a protein secreted by the liver that binds to apoB-lipoprotein particle and marks the LDLR for degradation on endocytosis. ${ }^{119}$ Adenoviral delivery of CRISPR/Cas9 into mice was very efficient in reducing PCSK9 and cholesterol in the blood, when $\sim 50 \%$ editing was achieved in the liver. ${ }^{120}$ Equally impressive results have been seen with AAV-mediated delivery with the $S$. aureus CRISPR system. ${ }^{36}$ Successful editing has also been achieved in human liver chimaeric mice, ${ }^{121}$ demonstrating the feasibility of targeting the human PCSK9 gene in hepatocytes. Although the benefits of a 'one-time treatment' are obvious and substantial in terms of sustained LDL lowering and patient compliance, it seems unlikely that these therapies will replace statins and PCSK9 inhibitors, which can be withdrawn at will if an adverse event should arise. Still many other proteins that are not currently targetable with small molecule inhibitors may also be candidates for substrate reduction.

\section{Metabolic pathway reprogramming}

Our group recently introduced a novel concept called metabolic pathway reprogramming ${ }^{50}$ (figure 3). This therapeutic approach can be applied to inborn errors of metabolism to avoid accumulation of toxic metabolites. Using this strategy, metabolic substrates can be rerouted to non-toxic avenues by inhibiting disease-associated genes instead of editing the mutated gene. As mentioned above, in a proof-of-concept study we converted HT-I ( $f a b^{-/-}$deficiency) into type III (HT-III) by deleting the $h p d$ gene. HT-III is a much more benign condition and in mice, we showed that double mutated hepatocytes $\left(f a h^{-/-} / h p d^{-1-}\right)$ have a growth advantage and eventually repopulate the whole liver. The reprogramming rescued both viability in the mice and all related symptoms associated with HT-I.

Tyrosinemia is currently treated by nitisinone, ${ }^{122}$ a small molecule inhibitor of HPD. However, the pharmacological block is incomplete, and although nitisinone reduces the risk of HT-I patients developing HCC, the incidence of this cancer is still significantly greater in this patient group. ${ }^{123}{ }^{124} \mathrm{fab}^{-/-}$mice treated with nitisinone also suffer an increased risk of HCC, ${ }^{125}$ but this risk disappears when the mice are crossed with $h p d^{-/-}$ (HT-III) mice. ${ }^{126}$ If metabolic pathway reprogramming for HT-I can eliminate the elevated risk of HCC, it might become a realistic alternative to nitisinone that could offer lasting correction. Metabolic pathway reprogramming should be applicable to many other inborn errors of metabolism, where there are currently no therapeutic options. These experimental therapies will take time to develop, and require a detailed knowledge of the pathway to be targeted.

\section{CRISPR/Cas9-mediated correction of mutated genes}

The most obvious therapeutic use for CRISPR/Cas9 is in correcting a mutated gene. Correcting a mutation by HDR necessitates a template with the wild-type sequence. The sister chromatid can serve as such a template and intragenic recombination has been described in many species. In humans, however, the evidence for intragenic recombination in somatic cells is poor ${ }^{127}$ and clonal expansion in the liver of HT-I patients cannot be explained by this phenomenon. ${ }^{128} 129$

The requirement of an additional element, whether singlestranded oligonucleotide or double-stranded DNA, complicates the approach from both a design and delivery standpoint. As mentioned previously, the more efficient NHEJ will compete with HDR in every cell. The efficiency of HDR is dependent on cell type and cell cycle, ${ }^{39}$ but varies considerably between different mammalian species, which is strikingly evident when comparing HDR in mouse and human embryonic stem cells. ${ }^{130}$ Therefore, we will have to await studies in primary human hepatocytes to reasonably assess the chances of success with CRISPR/Cas9-mediated HDR.

The first few studies demonstrating the feasibility of CRISPR/ Cas9-mediated gene corrections in the murine liver were encouraging. ${ }^{63-65}$ Nevertheless, the efficacy of gene correction in these studies was low. Additionally, all three studies were performed in the setting of increased mitotic rates, either in the expanding neonatal liver in the spf $f^{a s h}$ mice $^{63}$ or in adult $f a b^{-/}$ mice with constantly proliferating hepatocytes. ${ }^{64}{ }^{65}$ Therefore, genetic mutations detected shortly after birth or inborn errors of metabolism with liver regeneration such as HT-I are the best candidates for such an approach. Conceptually, however, almost all genetic liver diseases could be cured using this approach if it was possible to increase the rate of HDR by proliferation or other means (see section 'Translational challenges of CRISPR/ Cas9 genome editing'). Even if we were able to increase the rate of HDR significantly, this approach to gene correction would still likely introduce deleterious new mutations in the uncorrected alleles.

Although our focus here is on hepatocytes, the above clinical strategies could potentially be applied to disorders of nonparenchymal liver cells such as inhibiting cirroghenic factors of myofibroblasts or correcting factor VIII (haemophilia A) in liver endothelial cells.

\section{Translational challenges of CRISPR/Cas9 genome editing Delivery}

Delivery is a challenge for virtually every macromolecular therapy. Efficient hepatic genome editing will also depend on efficient and safe delivery of CRISPR/Cas9 components into the liver. While nanoparticles have shown promising results for transient expression of Cas9 or sgRNA in the liver, ${ }^{64} 131132$ these 
agents lag behind viral vectors in their clinical development. AAV vectors have been used successfully in over 120 clinical trials to date $^{133} 134$ (http://www.clinicaltrials.gov), with only little vectorrelated toxicity or adverse events. Liver-directed AAV vectors are in late stage clinical trials to treat haemophilia B (factor IX deficiency), and considerable success has already been achieved with AAV2, ${ }^{135}$ and AAV8-based vectors. ${ }^{136}{ }^{137}$ However, these clinical trials report a moderate dose-dependent immune response against the viral capsid. Therefore, reducing the dose with a more efficient AAV would be desirable.

The tropism and transduction efficiency of AAV is capsid (serotype)-dependent. ${ }^{138}$ Many AAV serotypes have been isolated from adenoviral stocks, non-human primates, or humans, and many of them evaluated in mice, dogs and non-human primates for liver-directed gene transfer. ${ }^{139-141}$ However, before extrapolating results from animal studies to humans for gene therapy, potential differences in uptake, delivery to the nucleus, uncoating, second strand synthesis of the recombinant genome and expression of the transgene must be considered. Humanised FRG mice ${ }^{111} 142143$ provide a unique in vivo platform to further evaluate candidate AAV serotypes for transduction efficiency of human hepatocytes. Several groups have recently published their first results using this animal model, ${ }^{144-147}$ but not all groups used the same set of serotypes. Additional experimental differences make it difficult to compare between these studies. One fundamental challenge with these studies is accounting for the effects of residual murine hepatocytes, which are more easily transduced by numerous serotypes than human cells. Better model systems and approaches are needed to find the best AAV serotypes for delivery to the human liver.

There are several remaining clinical hurdles for AAV gene therapy, including avoiding pre-existing neutralising antibodies to $\mathrm{AAV}^{135}{ }^{148}$ managing the immune responses to the capsid and transgene product, ${ }^{149}$ and the possibility of loss of episomal AAV genomes to cell division. While these challenges are more significant for classical gene therapy using a gene addition approach, CRISPR/Cas9 genome editing does not require sustained expression. Moreover, as we and others have shown in mouse models, successful genome editing in the liver can be achieved after only a few days. 50 63-65

Despite significant early setbacks, adenovirus may still be the best alternative to AAV for liver-directed gene therapy. Adenovirus is known to elicit a strong immune response in both mice and humans ${ }^{3}{ }^{150}$ and most humans have immunological memory from previous exposure. ${ }^{151}$ Helper-dependent adenovirus, so-called 'gutless' adenoviruses, have a better safety profile and retain excellent transduction efficiency of hepatocytes in experimental animal models, ${ }^{152}$ but have not been clinically developed for use in human livers. Herpes and retroviral vectors have a poor tropism for hepatocytes and the latter requires cell division. Similarly, lentiviral vectors, even when pseudotyped with vesicular stomatitis virus glycoprotein, are usually absorbed by non-parenchymal cells in the liver. ${ }^{153}$

In summary, choosing a safe and efficient delivery method for CRISPR/Cas9 applications is challenging, particularly when strategies with different macromolecular components (protein, DNA or RNA) are combined.

\section{Genotoxicity}

The potential genotoxicity of CRISPR/Cas9 and the vectors used for its delivery is a key issue that must be addressed prior to clinical translation. Cas9 cuts DNA only at sites that are recognised by its cognate sgRNA, and has no inherent nuclease activity in the absence of a sgRNA. ${ }^{154}$ However, base pairing between the sgRNA and the target site need not be perfect, and cleavage can still occur at sites with multiple mismatches. ${ }^{91} 155$ Considerable computational efforts have been undertaken to improve sgRNA design, as well as predict the most likely offtarget sites in the genome. ${ }^{156} 157$ While there are positions in the sgRNA/DNA duplex that are unfavourable to Cas9 activity, there are no universal rules that can be applied to ensure complete specificity. Validation and testing of sgRNA must ultimately be performed empirically.

Thus far, CRISPR/Cas9 seems to have a relatively low frequency of off-target mutagenesis in the liver with the $S$. pyogenes system. Viral delivery of SpCas9 with adenovirus produced no detectable mutagenesis of top predicted off-target sites in normal mice ${ }^{120}$ or human liver chimaeric animals. ${ }^{121} \mathrm{We}$ detected off-target mutagenesis of $2.8 \%$ in one of the 30 predicted sites using CRISPR/Cas9 to correct HT-I in the liver. ${ }^{50}$ Most recently, we used AAV vectors for sustained expression of sgRNAs in an SpCas9 transgenic mouse model and observed an off-target $5 \%$ mutagenesis rate with an sgRNA that had an on-target efficiency of $54 \% .{ }^{67}$ Ran et al identified a Cas 9 ortho$\log$ from $S$. aureus that is small enough to be delivered within AAV vectors. This ortholog of Cas9 appears to be highly efficient in the liver, and more specific than the commonly used $S$. pyogenes version. ${ }^{36}$ Two studies have used the S. aureus Cas9 system in the murine liver, and neither observed detectable offtarget mutagenesis at predicted sites; ${ }^{36}{ }^{63}$ however, more extensive genomic analyses were not performed on the livers. ${ }^{158} 159$

While the overall specificity of CRISPR/Cas9 for liver-directed genome editing looks promising, it should be noted that more robust genome wide and unbiased methods to assess specificity in human hepatocytes are needed. As shown in murine CRISPR/ Cas9 cancer models, even rare events disrupting tumour suppressor genes can trigger hepatocellular cancers. ${ }^{60-62}$

In addition to CRISPR/Cas9 itself, genotoxicity due to insertional mutagenesis of viral vectors should be considered. Adenovirus would seem to pose little risk since genomic integration in the liver is believed to be rare ${ }^{160}$ while the significance of insertional mutagenesis of AAV is controversial. Several groups have demonstrated that delivery of AAV to neonatal mice can cause $\mathrm{HCC}^{161}$ due to insertion at the murine Rian locus, ${ }^{162}{ }^{163}$ which is permissive to integration only early in development. ${ }^{162}$ In contrast, high-dose delivery of AAV vectors to adult mice generally does not increase the incidence of HCC, ${ }^{164} 165$ where integration events are estimated to occur in $\sim 1 / 588$ hepatocytes. ${ }^{165}$

We know far less about the risks of AAV integration in the human setting. In 2015, Nault et al published a study reporting integrations of wild-type AAV2 genomes in liver biopsies from patients with HCC. ${ }^{166}$ The frequency of integration events was paradoxically lower in the adjacent normal tissue relative to the tumour. Nonetheless, AAV integrations were found to expand clonally in several well-established proto-oncogenes known to underlie HCC driven by HBV infection (CCNA2, CCNE1, KMT2B, TERT). There has been only one study investigating rAAV integration in subjects receiving liver-directed gene therapy, where three human liver biopsies were examined. ${ }^{167}$ Integration events were rare $(<0.1 \%)$, randomly distributed and not detected at any known HCC hotspots. Given the lack of any epidemiological relationship between $\mathrm{AAV}$ infection and HCC, it seems unlikely that AAV will pose an inherent cancer risk, but this risk may also depend on the nature of the genetic cargo and activity of the transgene.

Most recently, we discovered integration of short Inverted Terminal Repeats (ITR) sequences at a DSB generated by 
CRISPR/Cas9 with AAV vectors in mouse liver. ${ }^{67}$ DSBs are known to promote AAV integration into the genome in cultured cells, ${ }^{168}$ and in the context of genome editing nucleases this is an even more important consideration. Advances in sequencing technology will continue to improve our ability to detect rare and potentially genotoxic events, and it will be important for the field to adopt uniform procedures to assess the risk of offtarget genome editing and vector integration. These risks must be weighed against the tremendous potential benefit to patients.

\section{Immunogenicity}

The majority of current clinical AAV applications seek to correct genes using constitutive promoters that may be more or less active than the endogenous promoter for that particular protein. This strategy makes protein expression difficult to regulate, and risks adverse events due to immune responses or unfavourable biological effects of excess transgene. Most importantly, gene addition relies on sustained expression of the transgene to achieve the therapeutic effect, while genome engineering only requires a small window of expression to accomplish the desired genomic alteration. From then on, the changes are genetically encoded and passed down to daughter cells.

One challenge in using CRISPR/Cas9 is the possibility that bacterial Cas9 will cause an unfavourable or prohibitive immune response in humans. Recent work using S. pyogenes Cas9 to delete the tumour suppressor Pten in the liver described the development of Cas9-specific antibodies and IL-2 secretion from Cas9-primed splenocytes. ${ }^{169}$ Of note, the Cas9 was delivered by adenoviral vectors, which are known to elicit an immune response and might have served as adjuvant. ${ }^{170}$ Another similar study, using adenovirus and CRISPR/Cas9 in the liver observed that Cas9 and the viral genome were massively reduced (1\%) 90 days after injection. ${ }^{171}$ In contrast to previous study, the immune response was not analysed. Interestingly, both studies report long-term persistence of highlevel genome editing on their target genes, in the case of Pten with the expected hepatomegaly and nonalcoholic steatohepatitis-like symptoms. Although it could be argued that Pten deletion might lead to a selective advantage and bias the results, these studies demonstrate that an immune response may not necessarily pose a problem for genome engineering.

The question remains how this immune response can be reduced and managed in the patient setting. A less immunogenic delivery method could help, but considering the fairly quick process of genome editing and the immunogenicity of bacterial proteins, it might be worth considering short-term immune suppression on injection. The road to clinical application is long and the bacterial kingdom is enormous, so there is plenty of time to find new and hopefully less immunogenic variants of the Cas9 protein or additional programmable nucleases from other species. ${ }^{172}{ }^{173}$ Potentially, gene therapy vectors could also be designed as self-deleting vectors harbouring one or several sgRNA target sites.

In the case of gene correction (see section 'Medical applications for CRISPR/Cas9 in the liver'), additional and likely longterm immune response might be triggered. However, we speculate that gene correction with proper regulation may be less immunogenic than strong transgenic expression from a gene therapy vector.

\section{Promoting HDR}

One major hurdle for gene correction strategies (see section 'Medical applications for CRISPR/Cas9 in the liver') for the liver is that active cell division is required for HDR. As the liver is largely quiescent organ under normal and even pathological circumstances, improved efficiency for HDR events must be achieved in order for CRISPR/Cas9-mediated HDR to be a viable possibility for liver repair studies.

Several groups have used small molecule inhibitors to block components of the NHEJ pathway in vitro and in vivo. ${ }^{174-178}$ Inhibiting DSB repair is a common strategy used in chemotherapy. However, excessive inhibition of DSB repair would impair normal cell repair processes, resulting in cell cycle arrest or apoptosis. Recently, it has also been shown that regions of heterochromatin in the DNA are difficult for Cas9 to target, and that these regions are edited with much lower efficiency than regions of euchromatin. ${ }^{179}$ This decreased efficiency could negatively impact the already low-efficiency HDR, and could hinder our ability to target genes that have a fluid chromatin state.

A potential alternative strategy to increase HDR is to use a novel CRISPR-Cas effector molecule Cpf1. ${ }^{172} \mathrm{Cpf1}$ is an enzyme that generates staggered $5^{\prime}$ prime overhang DSB rather than the blunt DSB created by Cas9. Cpf1 also cuts distal to the PAM, potentially allowing for more cycles of cutting and opportunities for HDR before the sgRNA-binding site is lost to NHEJ mutations. Using this enzyme could increase the rate of HDR, although currently there is not enough experience with Cpf1 to support its theoretical utility.

For now, the most efficient strategy, as demonstrated in mice, ${ }^{179}$ is correct genes in the immature, ideally neonatal liver, where there is more proliferation. An alternative is to choose therapeutic targets with a proliferation phenotype such as HT-I. Partial hepatectomy is also a theoretical possibility to make hepatocytes proliferate in animal models, but this strategy is unlikely to be beneficial in a clinical setting.

\section{SUMMARY}

More than 40 years ago, the first restriction enzyme was isolated and enabled development of modern molecular biology. Now CRISPR/Cas9 genome engineering takes the next step and enables even more precise genetic manipulation in living cells and organisms. Although it has the advantage that there is no need for sustained gene expression-a major limitation of traditional gene therapy (gene addition) - there is still concern about specificity and off-target cutting, which could result in genetic alterations that lead to cancer or other problems.

The CRISPR/Cas9 technology has rapidly conquered laboratories across the world and has generated a huge body of research, including the first preclinical therapeutic studies. Progress has already been made in animal models of ornithine transcarbamylase deficiency and HT, and work treating other inborn errors of metabolism is not far behind. We believe that liver disorders are particularly amenable to CRISPR/Cas9 genome editing and that the first clinical applications are close at hand.

Acknowledgements We would like to thank Paul Overbeek, Vicky Brandt and Catherine Gillespie for careful review of the manuscript. The authors apologise to those authors whose work was not cited owing to space limitations. K-DB is supported by the National Heart Lung and Blood Institute (NHLBI) grant R01HL134510, the Texas Hepatocellular Carcinoma Consortium (THCCC) (CPRIT \#RP150587) and the Diana Helis Henry and Adrienne Helis Malvin Medical Research WRL is supported by the NHLBI grant HL132840, FPP was supported by T32HL092332 and KEJ by T32HL07676.

Contributors All authors have contributed, written and accepted final version of the manuscript.

Funding National Heart and Lung Institute (HL132840, R01HL134510, T32HL07676 and T32HL092332) and Cancer Prevention and Research Institute of Texas (RP150587). 
Competing interests None declared.

Provenance and peer review Commissioned; internally peer reviewed.

\section{REFERENCES}

1 Crystal RG. Transfer of genes to humans: early lessons and obstacles to success. Science 1995;270:404-10.

2 Blaese RM, Culver KW, Miller AD, et al. T lymphocyte-directed gene therapy for ADA- SCID: initial trial results after 4 years. Science 1995;270:475-80.

3 Raper SE, Chirmule N, Lee FS, et al. Fatal systemic inflammatory response syndrome in a ornithine transcarbamylase deficient patient following adenoviral gene transfer. Mol Genet Metab 2003;80:148-58.

4 Hacein-Bey-Abina S, Von Kalle C, Schmidt M, et al. LMO2-associated clonal T cell proliferation in two patients after gene therapy for SCID-X1. Science 2003:302:415-19.

5 Knolle PA, Gerken G. Local control of the immune response in the liver. Immunol Rev 2000;174:21-34.

6 Ishino $Y$, Shinagawa H, Makino K, et al. Nucleotide sequence of the iap gene, responsible for alkaline phosphatase isozyme conversion in Escherichia coli, and identification of the gene product. J Bacterio/ 1987;169:5429-33.

7 Jansen $R$, Embden JD, Gaastra W, et al. Identification of genes that are associated with DNA repeats in prokaryotes. Mol Microbiol 2002;43:1565-75.

8 Bolotin A, Quinquis B, Sorokin A, et al. Clustered regularly interspaced short palindrome repeats (CRISPRs) have spacers of extrachromosomal origin. Microbiology 2005; 151:2551-61

9 Mojica FJ, Díez-Villaseñor C, Garcia-Martinez J, et al. Intervening sequences of regularly spaced prokaryotic repeats derive from foreign genetic elements. $J \mathrm{Mol}$ Evol 2005:60:174-82.

10 Pourcel C, Salvignol G, Vergnaud G. CRISPR elements in Yersinia pestis acquire new repeats by preferential uptake of bacteriophage DNA, and provide additional tools for evolutionary studies. Microbiology 2005;151:653-63.

11 Barrangou R, Fremaux C, Deveau $H$, et al. CRISPR provides acquired resistance against viruses in prokaryotes. Science 2007;315:1709-12.

12 Makarova KS, Grishin NV, Shabalina SA, et al. A putative RNA-interference-based immune system in prokaryotes: computational analysis of the predicted enzymatic machinery, functional analogies with eukaryotic RNAi, and hypothetical mechanisms of action. Biol Direct 2006;1:7.

13 Sapranauskas R, Gasiunas G, Fremaux C, et al. The Streptococcus thermophilus CRISPR/Cas system provides immunity in Escherichia coli. Nucleic Acids Res 2011;39:9275-82.

14 Deltcheva E, Chylinski K, Sharma CM, et al. CRISPR RNA maturation by trans-encoded small RNA and host factor RNase III. Nature 2011;471: 602-7.

15 Cho SW, Kim S, Kim JM, et al. Targeted genome engineering in human cells with the Cas9 RNA-guided endonuclease. Nat Biotechno/ 2013;31:230-2.

16 Jinek $M$, East $A$, Cheng $A$, et al. RNA-programmed genome editing in human cells. Elife 2013;2:e00471.

17 Mali P, Yang L, Esvelt KM, et al. RNA-guided human genome engineering via Cas9. Science 2013;339:823-6.

18 Cong $\mathrm{L}$, Ran FA, Cox D, et al. Multiplex genome engineering using CRISPR/Cas systems. Science 2013;339:819-23.

19 Brouns SJ, Jore MM, Lundgren $M$, et al. Small CRISPR RNAs guide antiviral defense in prokaryotes. Science 2008;321:960-4.

20 Mojica FJ, Diez-Villasenor C, Garcia-Martinez J, et al. Short motif sequences determine the targets of the prokaryotic CRISPR defence system. Microbiology 2009; 155:733-40.

21 Deveau H, Barrangou R, Garneau JE, et al. Phage response to CRISPR-encoded resistance in Streptococcus thermophilus. J Bacteriol 2008;190:1390-400.

22 Jinek M, Chylinski K, Fonfara I, et al. A programmable dual-RNA-guided DNA endonuclease in adaptive bacterial immunity. Science 2012;337:816-21.

23 Sternberg SH, LaFrance B, Kaplan M, et al. Conformational control of DNA target cleavage by CRISPR-Cas9. Nature 2015;527:110-13

24 Xu X, Duan D, Chen SJ. CRISPR-Cas9 cleavage efficiency correlates strongly with target-sgRNA folding stability: from physical mechanism to off-target assessment. Sci Rep 2017;7:143.

25 Singh D, Sternberg SH, Fei J, et al. Real-time observation of DNA recognition and rejection by the RNA-guided endonuclease Cas9. Nat Commun 2016;7:12778.

26 Garneau JE, Dupuis ME, Villion M, et al. The CRISPR/Cas bacterial immune system cleaves bacteriophage and plasmid DNA. Nature 2010;468:67-71.

27 Ran FA, Hsu PD, Wright J, et al. Genome engineering using the CRISPR-Cas9 system. Nat Protoc 2013;8:2281-308

28 Mali P, Aach J, Stranges PB, et al. CAS9 transcriptional activators for target specificity screening and paired nickases for cooperative genome engineering. Nat Biotechnol 2013:31:833-8.

29 Maeder ML, Linder SJ, Cascio VM, et al. CRISPR RNA-guided activation of endogenous human genes. Nat Methods 2013;10:977-9.

30 Perez-Pinera P, Kocak DD, Vockley CM, et al. RNA-guided gene activation by CRISPR-Cas9-based transcription factors. Nat Methods 2013;10:973-6.
31 Gilbert LA, Larson MH, Morsut L, et al. CRISPR-mediated modular RNA-guided regulation of transcription in eukaryotes. Cell 2013;154:442-51.

32 Xu X, Tao Y, Gao X, et al. A CRISPR-based approach for targeted DNA demethylation. Cell Discov 2016;2:16009.

33 McDonald JI, Celik H, Rois LE, et al. Reprogrammable CRISPR/Cas9-based system for inducing site-specific DNA methylation. Biol Open 2016:5:866-74.

34 Hilton IB, D'Ippolito AM, Vockley CM, et al. Epigenome editing by a CRISPR-Cas9-based acetyltransferase activates genes from promoters and enhancers. Nat Biotechnol 2015;33:510-17.

35 Kearns NA, Pham $H$, Tabak $B$, et al. Functional annotation of native enhancers with a Cas9-histone demethylase fusion. Nat Methods 2015;12:401-3.

36 Ran FA, Cong L, Yan WX, et al. In vivo genome editing using Staphylococcus aureus Cas9. Nature 2015:520:186-91.

37 Kleinstiver BP, Prew MS, Tsai SQ, et al. Broadening the targeting range of Staphylococcus aureus CRISPR-Cas9 by modifying PAM recognition. Nat Biotechnol 2015;33:1293-8.

38 Kleinstiver BP, Prew MS, Tsai SQ, et al. Engineered CRISPR-Cas9 nucleases with altered PAM specificities. Nature 2015;523:481-5.

39 Mao Z, Bozzella M, Seluanov A, et al. DNA repair by nonhomologous end joining and homologous recombination during cell cycle in human cells. Cell Cycle 2008;7:2902-6.

40 Branzei D, Foiani M. Regulation of DNA repair throughout the cell cycle. Nat Rev Mol Cell Biol 2008;9:297-308.

41 Rothkamm K, Krüger I, Thompson LH, et al. Pathways of DNA double-strand break repair during the mammalian cell cycle. Mol Cell Biol 2003;23:5706-15.

42 Symington LS, Gautier J. Double-strand break end resection and repair pathway choice. Annu Rev Genet 2011;45:247-71.

43 Jin C, Yuan FL, Gu YL, et al. Over-expression of ASIC1a promotes proliferation via activation of the beta-catenin/LEF-TCF axis and is associated with disease outcome in liver cancer. Oncotarget 2017;8:25977-88.

44 Pott LL, Hagemann S, Reis $\mathrm{H}$, et al. Eukaryotic elongation factor 2 is a prognostic marker and its kinase a potential therapeutic target in HCC. Oncotarget 2017:8:11950-62.

45 Borger DR, Tanabe KK, Fan KC, et al. Frequent mutation of isocitrate dehydrogenase (IDH) 1 and IDH2 in cholangiocarcinoma identified through broad-based tumor genotyping. Oncologist 2012;17:72-9.

46 Ye J, Gu Y, Zhang F, et al. IDH1 deficiency attenuates gluconeogenesis in mouse liver by impairing amino acid utilization. Proc Natl Acad Sci USA 2017:114:292-7.

47 Iwagami Y, Huang CK, Olsen MJ, et al. Aspartate $\beta$-hydroxylase modulates cellular senescence through glycogen synthase kinase $3 \beta$ in hepatocellular carcinoma. Hepatology 2016;63:1213-26.

48 Yang $\mathrm{H}$, Wang $\mathrm{H}$, Shivalila $\mathrm{CS}$, et al. One-step generation of mice carrying reporter and conditional alleles by CRISPR/Cas-mediated genome engineering. Cell 2013;154:1370-9.

49 Canver MC, Bauer DE, Dass A, et al. Characterization of genomic deletion efficiency mediated by clustered regularly interspaced palindromic repeats (CRISPR)/Cas9 nuclease system in mammalian cells. J Biol Chem 2014;289:21312-24.

50 Pankowicz FP, Barzi M, Legras $X$, et al. Reprogramming metabolic pathways in vivo with CRISPR/Cas9 genome editing to treat hereditary tyrosinaemia. Nat Commun 2016;7:12642.

51 Wang D, Papp AC, Sun X. Functional characterization of CYP2D6 enhancer polymorphisms. Hum Mol Genet 2015;24:1556-62.

52 Zhu P, Wang Y, He L, et al. ZIC2-dependent OCT4 activation drives self-renewal of human liver cancer stem cells. J Clin Invest 2015;125:3795-808.

53 Li S, Xue H, Wu J, et al. Human induced pluripotent stem cell NEUROG2 dual knockin reporter lines generated by the CRISPR/Cas9 system. Stem Cells Dev 2015;24:2925-42

54 Ratz M, Testa I, Hell SW, et al. CRISPR/Cas9-mediated endogenous protein tagging for RESOLFT super-resolution microscopy of living human cells. Sci Rep 2015;5:9592.

55 Tian L, Deshmukh A, Ye Z, et al. Efficient and controlled generation of 2D and 3D bile duct tissue from human pluripotent stem cell-derived spheroids. Stem Cell Rev 2016:12:500-8.

56 Lee PC, Truong B, Vega-Crespo A, et al. Restoring ureagenesis in hepatocytes by CRISPR/Cas9-mediated genomic addition to arginase-deficient induced pluripotent stem cells. Mol Ther Nucleic Acids 2016;5:e394.

57 Luizon MR, Eckalbar WL, Wang Y, et al. Genomic characterization of metformin hepatic response. PLoS Genet 2016;12:e1006449.

58 Liu F, Song Y, Liu D. Hydrodynamics-based transfection in animals by systemic administration of plasmid DNA. Gene Ther 1999;6:1258-66.

59 Zhang G, Budker V, Wolff JA. High levels of foreign gene expression in hepatocytes after tail vein injections of naked plasmid DNA. Hum Gene Ther 1999:10:1735-7.

60 Xue W, Chen S, Yin H, et al. CRISPR-mediated direct mutation of cancer genes in the mouse liver. Nature 2014:514:380-4.

61 Weber J, Öllinger R, Friedrich $M$, et al. CRISPR/Cas9 somatic multiplex-mutagenesis for high-throughput functional cancer genomics in mice. Proc Natl Acad Sci USA 2015;112:13982-7. 
62 Song CQ, Li Y, Mou H, et al. Genome-wide CRISPR screen identifies regulators of mitogen-activated protein kinase as suppressors of liver tumors in mice. Gastroenterology 2017;152:1161-73.e1.

63 Yang Y, Wang L, Bell P, et al. A dual AAV system enables the Cas9-mediated correction of a metabolic liver disease in newborn mice. Nat Biotechnol 2016:34:334-8.

64 Yin H, Song CQ, Dorkin JR, et al. Therapeutic genome editing by combined viral and non-viral delivery of CRISPR system components in vivo. Nat Biotechnol 2016;34:328-33.

65 Yin $\mathrm{H}$, Xue W, Chen $\mathrm{S}$, et al. Genome editing with Cas9 in adult mice corrects a disease mutation and phenotype. Nat Biotechnol 2014;32:551-3.

66 Liang WC, Liang PP, Wong CW, et al. CRISPR/Cas9 technology targeting fas gene protects mice from concanavalin-A induced fulminant hepatic failure. J Cell Biochem 2017;118:530-6.

67 Jarrett $\mathrm{KE}$, Lee $\mathrm{CM}$, Yeh YH, et al. Somatic genome editing with CRISPR/Cas9 generates and corrects a metabolic disease. Sci Rep 2017;7:44624.

68 Capecchi MR. Gene targeting in mice: functional analysis of the mammalian genome for the twenty-first century. Nat Rev Genet 2005;6:507-12.

69 Hwang WY, Fu Y, Reyon D, et al. Efficient genome editing in zebrafish using a CRISPR-Cas system. Nat Biotechnol 2013;31:227-9.

70 Jao LE, Wente SR, Chen W. Efficient multiplex biallelic zebrafish genome editing using a CRISPR nuclease system. Proc Natl Acad Sci USA 2013;110:13904-9.

71 Chang N, Sun C, Gao L, et al. Genome editing with RNA-guided Cas9 nuclease in zebrafish embryos. Cell Res 2013;23:465-72.

72 Wang $H$, Yang $H$, Shivalila CS, et al. One-step generation of mice carrying mutations in multiple genes by CRISPR/Cas-mediated genome engineering. Cell 2013;153:910-18.

73 Shen $\mathrm{B}$, Zhang J, Wu H, et al. Generation of gene-modified mice via Cas9/ RNA-mediated gene targeting. Cell Res 2013;23:720-3.

74 Li W, Teng F, Li T, et al. Simultaneous generation and germline transmission of multiple gene mutations in rat using CRISPR-Cas systems. Nat Biotechnol 2013:31:684-6.

75 Li D, Qiu Z, Shao Y, et al. Heritable gene targeting in the mouse and rat using a CRISPR-Cas system. Nat Biotechnol 2013;31:681-3.

76 Yang $\mathrm{D}, \mathrm{Xu}$ J, Zhu T, et al. Effective gene targeting in rabbits using RNA-guided Cas9 nucleases. J Mol Cell Biol 2014;6:97-9.

77 Michel M, Page-McCaw PS, Chen W, et al. Leptin signaling regulates glucose homeostasis, but not adipostasis, in the zebrafish. Proc Natl Acad Sci USA 2016;113:3084-9.

78 Yin L, Maddison LA, Li M, et al. Multiplex conditional mutagenesis using transgenic expression of Cas9 and sgRNAs. Genetics 2015;200:431-41.

79 Kozlitina J, Smagris E, Stender S, et al. Exome-wide association study identifies a TM6SF2 variant that confers susceptibility to nonalcoholic fatty liver disease. Nat Genet 2014;46:352-6.

80 Holmen OL, Zhang $\mathrm{H}$, Fan $\mathrm{Y}$, et al. Systematic evaluation of coding variation identifies a candidate causal variant in TM6SF2 influencing total cholesterol and myocardial infarction risk. Nat Genet 2014;46:345-51.

81 Fan Y, Lu H, Guo Y, et al. Hepatic transmembrane 6 superfamily member 2 regulates cholesterol metabolism in mice. Gastroenterology 2016;150:1208-18.

82 Nakagawa Y, Oikawa F, Mizuno S, et al. Hyperlipidemia and hepatitis in liver-specific CREB3L3 knockout mice generated using a one-step CRISPR/Cas9 system. Sci Rep 2016;6:27857.

83 Zhang L, Shao Y, Li L, et al. Efficient liver repopulation of transplanted hepatocyte prevents cirrhosis in a rat model of hereditary tyrosinemia type I. Sci Rep 2016:6:31460.

84 Wang X, Tang Y, Lu J, et al. Characterization of novel cytochrome P450 2E1 knockout rat model generated by CRISPR/Cas9. Biochem Pharmacol 2016;105:80-90.

85 Lu J, Shao Y, Qin X, et al. CRISPR knockout rat cytochrome P450 3A1/2 model for advancing drug metabolism and pharmacokinetics research. Sci Rep 2017:7:42922.

86 Barzi M, Pankowicz FP, Zorman B, et al. A novel humanized mouse lacking murine P450 oxidoreductase for studying human drug metabolism. Nat Commun 2017; in press.

87 Pham CT, Maclvor DM, Hug BA, et al. Long-range disruption of gene expression by a selectable marker cassette. Proc Natl Acad Sci USA 1996;93:13090-5.

88 Olson EN, Arnold HH, Rigby PW, et al. Know your neighbors: three phenotypes in null mutants of the myogenic bHLH gene MRF4. Cell 1996;85:1-4.

89 Lagor WR, Fields DW, Bauer RC, et al. Genetic manipulation of the ApoF/Stat2 locus supports an important role for type I interferon signaling in atherosclerosis. Atherosclerosis 2014;233:234-41.

90 Hsu PD, Scott DA, Weinstein JA, et al. DNA targeting specificity of RNA-guided Cas9 nucleases. Nat Biotechnol 2013;31:827-32.

91 Fu Y, Foden JA, Khayter $C$, et al. High-frequency off-target mutagenesis induced by CRISPR-Cas nucleases in human cells. Nat Biotechnol 2013;31:822-6.

92 Adams D, Samuel D, Goulon-Goeau C, et al. The course and prognostic factors of familial amyloid polyneuropathy after liver transplantation. Brain 2000;123: 1495-504
93 Hörnsten R, Wiklund U, Olofsson BO, et al. Liver transplantation does not prevent the development of life-threatening arrhythmia in familial amyloidotic polyneuropathy, Portuguese-type (ATTR Val30Met) patients. Transplantation 2004;78:112-16.

94 Yazaki M, Tokuda T, Nakamura A, et al. Cardiac amyloid in patients with familial amyloid polyneuropathy consists of abundant wild-type transthyretin. Biochem Biophys Res Commun 2000;274:702-6.

95 Honeyman JN, Simon EP, Robine N, et al. Detection of a recurrent DNAJB1-PRKACA chimeric transcript in fibrolamellar hepatocellular carcinoma. Science 2014:343:1010-4.

96 Kennedy EM, Kornepati AV, Goldstein M, et al. Inactivation of the human papillomavirus E6 or E7 gene in cervical carcinoma cells by using a bacterial CRISPR/Cas RNA-guided endonuclease. J Virol 2014:88:11965-72.

97 Zhen S, Hua L, Takahashi Y, et al. In vitro and in vivo growth suppression of human papillomavirus 16-positive cervical cancer cells by CRISPR/Cas9. Biochem Biophys Res Commun 2014;450:1422-6.

98 Yuen KS, Chan CP, Wong NH, et al. CRISPR/Cas9-mediated genome editing of Epstein-Barr virus in human cells. J Gen Virol 2015;96:626-36.

99 Wang J, Quake SR. RNA-guided endonuclease provides a therapeutic strategy to cure latent herpesviridae infection. Proc Natl Acad Sci USA 2014;111:13157-62.

100 Yan $\mathrm{H}$, Zhong G, Xu G, et al. Sodium taurocholate cotransporting polypeptide is a functional receptor for human hepatitis B and D virus. Elife 2012;1:e00049.

101 Lin SR, Yang HC, Kuo YT, et al. The CRISPR/Cas9 system facilitates clearance of the intrahepatic HBV templates in vivo. Mol Ther Nucleic Acids 2014;3:e186.

102 Kennedy EM, Bassit LC, Mueller H, et al. Suppression of hepatitis B virus DNA accumulation in chronically infected cells using a bacterial CRISPR/Cas RNA-guided DNA endonuclease. Virology 2015;476:196-205.

103 Dong C, Qu L, Wang H, et al. Targeting hepatitis B virus cccDNA by CRISPR/Cas9 nuclease efficiently inhibits viral replication. Antiviral Res 2015;118:110-17.

104 Jiang C, Mei M, Li B, et al. A non-viral CRISPR/Cas9 delivery system for therapeutically targeting HBV DNA and pcsk9 in vivo. Cell Res 2017;27:440-3.

105 Zhu W, Xie K, Xu Y, et al. CRISPR/Cas9 produces anti-hepatitis B virus effect in hepatoma cells and transgenic mouse. Virus Res 2016;217:125-32.

106 Karimova M, Beschorner N, Dammermann W, et al. CRISPR/Cas9 nickase-mediated disruption of hepatitis $B$ virus open reading frame $S$ and $X$. Sci Rep 2015;5:13734.

107 Ramanan V, Shlomai A, Cox DB, et al. CRISPR/Cas9 cleavage of viral DNA efficiently suppresses hepatitis B virus. Sci Rep 2015;5:10833.

108 Liu X, Hao R, Chen S, et al. Inhibition of hepatitis B virus by the CRISPR/Cas9 system via targeting the conserved regions of the viral genome. J Gen Virol 2015;96:2252-61.

109 Seeger C, Sohn JA. Targeting Hepatitis B Virus With CRISPR/Cas9. Mol Ther Nucleic Acids 2014;3:e216.

110 Qi Z, Li G, Hu H, et al. Recombinant covalently closed circular hepatitis B virus DNA induces prolonged viral persistence in immunocompetent mice. J Virol 2014;88:8045-56

111 Bissig KD, Wieland SF, Tran $\mathrm{P}$, et al. Human liver chimeric mice provide a model for hepatitis B and C virus infection and treatment. J Clin Invest 2010;120:924-30.

112 Dandri M, Burda MR, Török $E$, et al. Repopulation of mouse liver with human hepatocytes and in vivo infection with hepatitis B virus. Hepatology 2001;33:981-8

113 Price AA, Sampson TR, Ratner HK, et al. Cas9-mediated targeting of viral RNA in eukaryotic cells. Proc Natl Acad Sci USA 2015;112:6164-9.

114 Slijepcevic D, Kaufman C, Wichers CG, et al. Impaired uptake of conjugated bile acids and hepatitis $b$ virus pres 1 -binding in na(+) -taurocholate cotransporting polypeptide knockout mice. Hepatology 2015;62:207-19.

115 Rines AK, Sharabi K, Tavares CD, et al. Targeting hepatic glucose metabolism in the treatment of type 2 diabetes. Nat Rev Drug Discov 2016;15:786-804.

116 Osono Y, Woollett LA, Herz J, et al. Role of the low density lipoprotein receptor in the flux of cholesterol through the plasma and across the tissues of the mouse. J Clin Invest 1995;95:1124-32.

117 Brown MS, Goldstein JL. A receptor-mediated pathway for cholesterol homeostasis. Science 1986;232:34-47.

118 Duell PB, Santos RD, Kirwan BA, et al. Long-term mipomersen treatment is associated with a reduction in cardiovascular events in patients with familia hypercholesterolemia. J Clin Lipidol 2016;10:1011-21.

119 Horton JD, Cohen JC, Hobbs HH. Molecular biology of PCSK9: its role in LDL metabolism. Trends Biochem Sci 2007:32:71-7.

120 Ding Q, Strong A, Patel KM, et al. Permanent alteration of PCSK9 with in vivo CRISPR-Cas9 genome editing. Circ Res 2014;115:488-92.

121 Wang $X$, Raghavan A, Chen T, et al. CRISPR-Cas9 targeting of pcsk9 in human hepatocytes in vivo-brief report. Arterioscler Thromb Vasc Biol 2016;36:783-6.

122 Lindstedt S, Holme E, Lock EA, et al. Treatment of hereditary tyrosinaemia type I by inhibition of 4-hydroxyphenylpyruvate dioxygenase. Lancet 1992;340:813-17.

123 Holme E, Lindstedt S. Nontransplant treatment of tyrosinemia. Clin Liver Dis 2000;4:805-14.

124 Holme E, Lindstedt S. Tyrosinaemia type I and NTBC (2-(2-nitro-4-trifluoromethylbenzoyl)1,3-cyclohexanedione). J Inherit Metab Dis 1998;21:507-17. 
125 Grompe M, Lindstedt S, al-Dhalimy $\mathrm{M}$, et al. Pharmacological correction of neonatal lethal hepatic dysfunction in a murine model of hereditary tyrosinaemia type I. Nat Genet 1995;10:453-60.

126 Endo F, Kubo S, Awata H, et al. Complete rescue of lethal albino c14CoS mice by null mutation of 4-hydroxyphenylpyruvate dioxygenase and induction of apoptosis of hepatocytes in these mice by in vivo retrieval of the tyrosine catabolic pathway. J Biol Chem 1997;272:24426-32.

127 Hirschhorn R. In vivo reversion to normal of inherited mutations in humans. J Med Genet 2003;40:721-8.

128 Demers SI, Russo P, Lettre $\mathrm{F}$, et al. Frequent mutation reversion inversely correlates with clinical severity in a genetic liver disease, hereditary tyrosinemia. Hum Pathol 2003:34:1313-20.

129 Kvittingen EA, Rootwelt $H$, Berger $R$, et al. Self-induced correction of the genetic defect in tyrosinemia type I. J Clin Invest 1994;94:1657-61.

130 Zwaka TP, Thomson JA. Homologous recombination in human embryonic stem cells. Nat Biotechnol 2003;21:319-21.

131 Miller JB, Zhang S, Kos P, et al. Non-viral CRISPR/cas gene editing in vitro and in vivo enabled by synthetic nanoparticle co-delivery of Cas9 mRNA and sgRNA. Angew Chem Int Ed Engl 2017;56:1059-63.

132 Zuris JA, Thompson DB, Shu Y, et al. Cationic lipid-mediated delivery of proteins enables efficient protein-based genome editing in vitro and in vivo. Nat Biotechnol 2015;33:73-80.

133 Asokan A, Schaffer DV, Jude Samulski R. The AAV vector toolkit: poised at the clinical crossroads. Mol Ther 2012;20:699-708.

134 Mingozzi F, High KA. Therapeutic in vivo gene transfer for genetic disease using AAV: progress and challenges. Nat Rev Genet 2011;12:341-55.

135 Manno CS, Pierce GF, Arruda VR, et al. Successful transduction of liver in hemophilia by AAV-Factor IX and limitations imposed by the host immune response. Nat Med 2006;12:342-7.

136 Nathwani AC, Tuddenham EG, Rangarajan S, et al. Adenovirus-associated virus vector-mediated gene transfer in hemophilia B. N Engl J Med 2011;365: 2357-65.

137 Nathwani AC, Reiss UM, Tuddenham EG, et al. Long-term safety and efficacy of factor IX gene therapy in hemophilia B. N Engl J Med 2014;371:1994-2004.

138 Grimm D, Pandey K, Nakai $\mathrm{H}$, et al. Liver transduction with recombinant adeno-associated virus is primarily restricted by capsid serotype not vector genotype. J Virol 2006;80:426-39.

139 Bell P, Gao G, Haskins ME, et al. Evaluation of adeno-associated viral vectors for liver-directed gene transfer in dogs. Hum Gene Ther 2011;22:985-97.

140 Wang L, Calcedo R, Wang $H$, et al. The pleiotropic effects of natural AAV infections on liver-directed gene transfer in macaques. Mol Ther 2010;18:126-34.

141 Wang L, Wang H, Bell P, et al. Systematic evaluation of AAV vectors for liver directed gene transfer in murine models. Mol Ther 2010;18:118-25.

142 Bissig KD, Le TT, Woods NB, et al. Repopulation of adult and neonatal mice with human hepatocytes: a chimeric animal model. Proc Natl Acad Sci USA 2007;104:20507-11.

143 Azuma H, Paulk N, Ranade A, et al. Robust expansion of human hepatocytes in Fah(-/-)/Rag2(-/-)/II2rg(-/-) mice. Nat Biotechnol 2007;25:903-10.

144 Lisowski L, Dane AP, Chu K, et al. Selection and evaluation of clinically relevant AAV variants in a xenograft liver model. Nature 2014;506:382-6.

145 Bissig-Choisat B, Wang L, Legras $X$, et al. Development and rescue of human familial hypercholesterolaemia in a xenograft mouse model. Nat Commun 2015;6:7339.

146 Wang L, Bell P, Somanathan S, et al. Comparative study of liver gene transfer with AAV vectors based on natural and engineered AAV capsids. Mol Ther 2015;23:1877-87

147 Vercauteren $\mathrm{K}$, Hoffman BE, Zolotukhin I, et al. Superior in vivo transduction of human hepatocytes using engineered AAV3 capsid. Mol Ther 2016;24:1042-9.

148 Boutin S, Monteilhet $\mathrm{V}$, Veron $\mathrm{P}$, et al. Prevalence of serum IgG and neutralizing factors against adeno-associated virus (AAV) types 1, 2, 5, 6, 8, and 9 in the healthy population: implications for gene therapy using AAV vectors. Hum Gene Ther 2010;21:704-12

149 Arruda VR, Samelson-Jones BJ. Gene therapy for immune tolerance induction in hemophilia with inhibitors. J Thromb Haemost 2016;14:1121-34.

150 Muruve DA. The innate immune response to adenovirus vectors. Hum Gene Ther 2004:15:1157-66.

151 Wold WS, Toth K. Adenovirus vectors for gene therapy, vaccination and cancer gene therapy. Curr Gene Ther 2013;13:421-33.
152 Brunetti-Pierri N, Ng P. Helper-dependent adenoviral vectors for liver-directed gene therapy. Hum Mol Genet 2011;20:R7-13.

153 van Til NP, Markusic DM, van der Rijt $\mathrm{R}$, et al. Kupffer cells and not liver sinusoidal endothelial cells prevent lentiviral transduction of hepatocytes. $\mathrm{Mol}$ Ther 2005;11:26-34.

154 Tsai SQ, Joung JK. Defining and improving the genome-wide specificities of CRISPR-Cas9 nucleases. Nat Rev Genet 2016;17:300-12.

155 Lin Y, Cradick TJ, Brown MT, et al. CRISPR/Cas9 systems have off-target activity with insertions or deletions between target DNA and guide RNA sequences. Nucleic Acids Res 2014:42:7473-85.

156 Cradick TJ, Qiu P, Lee CM, et al. COSMID: a web-based tool for identifying and validating CRISPR/Cas off-target sites. Mol Ther Nucleic Acids 2014:3:e214.

157 Montague TG, Cruz JM, Gagnon JA, et al. CHOPCHOP: a CRISPR/Cas9 and TALEN web tool for genome editing. Nucleic Acids Res 2014;42:W401-7.

158 Kleinstiver BP, Pattanayak V, Prew MS, et al. High-fidelity CRISPR-Cas9 nucleases with no detectable genome-wide off-target effects. Nature 2016

159 Slaymaker IM, Gao L, Zetsche B, et al. Rationally engineered Cas9 nucleases with improved specificity. Science 2016;351:84-8.

160 Stephen SL, Montini E, Sivanandam VG, et al. Chromosomal integration of adenoviral vector DNA in vivo. J Virol 2010;84:9987-94.

161 Donsante A, Vogler C, Muzyczka N, et al. Observed incidence of tumorigenesis in long-term rodent studies of rAAV vectors. Gene Ther 2001;8:1343-6.

162 Chandler RJ, LaFave MC, Varshney GK, et al. Vector design influences hepatic genotoxicity after adeno-associated virus gene therapy. J Clin Invest 2015;125:870-80.

163 Donsante A, Miller DG, Li Y, et al. AAV vector integration sites in mouse hepatocellular carcinoma. Science 2007:317:477.

164 Bell P, Moscioni AD, McCarter RJ, et al. Analysis of tumors arising in male B6C3F1 mice with and without AAV vector delivery to liver. Mol Ther 2006; 14:34-44.

165 Li H, Malani N, Hamilton SR, et al. Assessing the potential for AAV vector genotoxicity in a murine model. Blood 2011;117:3311-19.

166 Nault JC, Datta S, Imbeaud S, et al. Recurrent AAV2-related insertional mutagenesis in human hepatocellular carcinomas. Nat Genet 2015;47: 1187-93.

167 Gil-Farina I, Fronza R, Kaeppel C, et al. Recombinant AAV integration is not associated with hepatic genotoxicity in nonhuman primates and patients. Mol Ther 2016:24:1100-5.

168 Miller DG, Petek LM, Russell DW. Adeno-associated virus vectors integrate at chromosome breakage sites. Nat Genet 2004;36:767-73.

169 Wang D, Mou H, Li S, et al. Adenovirus-mediated somatic genome editing of Pten by CRISPR/Cas9 in mouse liver in spite of Cas9-specific immune responses. Hum Gene Ther 2015:26:432-42.

170 Geutskens SB, van der Eb MM, Plomp AC, et al. Recombinant adenoviral vectors have adjuvant activity and stimulate $T$ cell responses against tumor cells. Gene Ther 2000;7:1410-16.

171 Cheng R, Peng J, Yan Y, et al. Efficient gene editing in adult mouse livers via adenoviral delivery of CRISPR/Cas9. FEBS Lett 2014;588:3954-8.

172 Burstein D, Harrington LB, Strutt SC, et al. New CRISPR-Cas systems from uncultivated microbes. Nature 2017;542:237-41.

173 Zetsche B, Gootenberg JS, Abudayyeh 00, et al. Cpf1 is a single RNA-guided endonuclease of a class 2 CRISPR-Cas system. Cell 2015;163:759-71.

174 Robert F, Barbeau M, Éthier S, et al. Pharmacological inhibition of DNA-PK stimulates Cas9-mediated genome editing. Genome Med 2015;7:93.

175 Vartak SV, Raghavan SC. Inhibition of nonhomologous end joining to increase the specificity of CRISPR/Cas9 genome editing. FEBS J 2015;282: 4289-94

176 Maruyama T, Dougan SK, Truttmann MC, et al. Increasing the efficiency of precise genome editing with CRISPR-Cas9 by inhibition of nonhomologous end joining. Nat Biotechnol 2015;33:538-42.

177 Yu C, Liu Y, Ma T, et al. Small molecules enhance CRISPR genome editing in pluripotent stem cells. Cell Stem Cell 2015:16:142-7.

178 Srivastava M, Nambiar M, Sharma S, et al. An inhibitor of nonhomologous end-joining abrogates double-strand break repair and impedes cancer progression. Cell 2012;151:1474-87.

179 Chen $X$, Rinsma M, Janssen JM, et al. Probing the impact of chromatin conformation on genome editing tools. Nucleic Acids Res 2016;44:6482-92. 